\section{Brazilian Journal \\ of Chemical Engineering}

ISSN 0104-6632

Printed in Brazil

www.abeq.org.br/bjche

\title{
MODELLING OF DISPLACEMENT WASHING OF PACKED BED OF FIBERS
}

\author{
S. Arora ${ }^{1 *}$ and F. Potůček ${ }^{2}$ \\ ${ }^{1}$ Department of Mathematics, Punjabi University, Phone: $+(91)$ 98152-85794, (M), \\ Fax: +(91) 175-3046189, Patiala, 147002, Punjab, India. \\ E-mail: shellyarora_25@yahoo.co.in \\ ${ }^{2}$ Department of Wood, Pulp and Paper, Faculty of Chemical Technology, University of Pardubice, \\ Phone: + (420) 466038016 (O), Fax: + (420) 466038039 , \\ Studentská 95, 53210 Pardubice, Czech Republic. \\ E-mail: frantisek.potucek@upce.cz
}

(Submitted: March 23, 2008 ; Revised: July 22, 2008 ; Accepted: August 29, 2008)

\begin{abstract}
The mechanism of displacement washing of packed bed of porous, compressible and cylindrical particles, e.g., fibers, is presented with the help of an axial dispersion model involving Peclet number $(P e)$ and Biot number $(B i)$. Bulk fluid concentration, intra-pore solute concentration and concentration of solute adsorbed on the fiber surface are taken to be the function of washing time and position in the bed. Concentration of solute adsorbed on the fiber surface and intra-pore solute concentration are related by a Langmuir adsorption isotherm. Experiments have been performed on pulp beds formed from unbeaten, unbleached kraft fibers. Displacement washing has been simulated using a laboratory washing cell. Model equations are solved by using orthogonal collocation on finite elements (OCFE). Model predicted values are compared with the experimental values and key industrial parameters such as displacement ratio and efficiency are expressed in terms of exit and average solute concentrations.

Keywords: Displacement Washing; Mathematical model; Peclet number; Biot number.
\end{abstract}

\section{INTRODUCTION}

The problem of heat and mass transfer during flow through packed beds of porous, compressible particles is of great interest in the field of chemical engineering and processing. For the past few decades, these problems have gained momentum in the field of mathematical modelling as well. A variety of models related to adsorption-desorption and diffusiondispersion phenomena (Raghvan and Ruthven, 1983; Al-Jabari et al., 1994; Sridhar et al., 1994; Eriksson et al., 1996; Potucek, 1997; Liu and Bhatia, 2001; Ding and Bhatia, 2003; Arora et al., 2006) have been developed. Bhatia (1987) and Liu and Bhatia (2001) have discussed radial diffusivity in gaseous form. Sharma and Yortsos (1987), and Immanuel et al. (2003) have considered the effective diffusion coefficient in porous media. Balasubramanian et al. (1997) have considered the radial dispersion in curved tubes. Ding and Bhatia (2003) have considered Knudsen and molecular diffusivity depending on the pore size of particle, whereas Arora et al. (2006a) have discussed pore diffusion in porous fibers.

In the present study, displacement washing of fibers that are porous in nature is presented through a dynamic model using Peclet number, $P e$, and Biot number, Bi. Peclet number is ratio of advection to dispersion, whereas the Biot number relates mass transfer resistances inside and on the surface of the body.

In the washing process, solute present in the irregular void channels of the bed, associated with diffusion-like dispersion, is removed by the introduction of a solvent. Due to the porous nature of

*To whom correspondence should be addressed 
the fibers, solute present in pores of the fibers diffuses out when it comes in contact with external fluid. Therefore, for packed beds of porous particles, the mechanism of axial dispersion in the continuous phase between particles and the concentration of fluid within and outside the particles play an important role. Due to adsorption of solute on the fiber surface, mass transfer takes place through the internal and external fiber surface and from the fiber surface to the external fluid.

\section{MODEL FORMULATION}

Basically there are two types of mechanisms related to fluid concentration in a packed bed of fibers. The first one is related to the transfer rate of material between fluid and fibers and is given explicitly by the equation:

$$
\frac{\partial \mathrm{d}}{\partial \mathrm{t}}=\mathrm{F}(\mathrm{c}, \mathrm{q})
$$

This type of isotherm has been discussed widely for both linear and non-linear isotherms. The second type of rate mechanism is one in which solid phase diffusion into the interior of the bed particles is an important rate step and the rate equation $F(c, q)$ is replaced by an integral equation. Details of the second type of rate mechanism are given elsewhere (Arora et al., 2006). In this paper, the first type of rate mechanism employs a Langmuir adsorption isotherm to study the displacement washing of fluid flow through the bed of packed fibers. Schematic representation of the isothermal system for a macroscopically uniform packed bed of porous fibers is discussed in Figure 1.

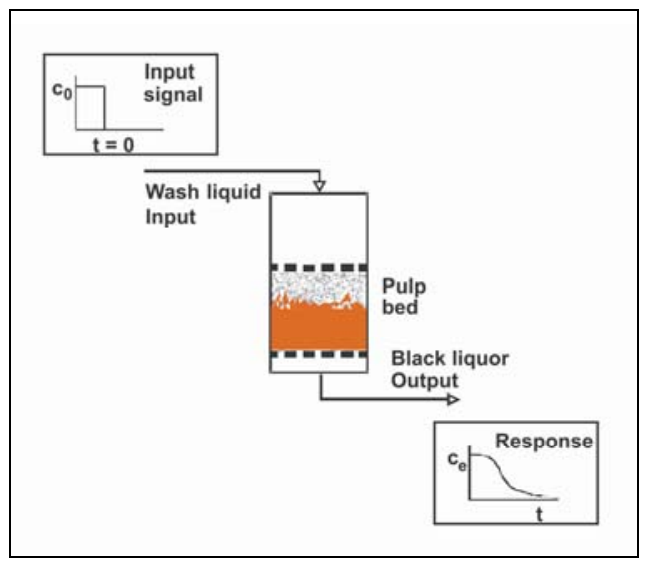

Figure 1: Schematic representation of the system of washing cell.
Flow of fluid through the bed is described by the bulk fluid concentration $c(z, t)$. The concentration of solute adsorbed on the fiber surface and the intrapore solute concentrations are described by $n(z, t)$ and $q(z, t)$, respectively. Fiber and bed porosities are described by $\beta$ and $\varepsilon$, respectively. Movement of solute within fiber pores is defined by Fick's law and dispersion in the fluid is defined by the axial dispersion coefficient $D_{L}$ which is independent of axial distance $L$. Mass transfer through the stagnant layer is controlled by the film resistance mass transfer coefficient $k_{f}$. The unsteady-state partial differential equations describing the behavior of fluid flow through the packed bed are given below:

\section{Model Equations for Particle Phase}

$$
\frac{\partial \mathrm{q}}{\partial \mathrm{t}}+\frac{1-\beta}{\beta} \frac{\partial \mathrm{n}}{\partial \mathrm{t}}=\frac{\mathrm{k}_{\mathrm{f}}}{\mathrm{KR}}(\mathrm{c}-\mathrm{q})
$$

\section{Adsorption Isotherm}

Langmuir type adsorption isotherm has been used to relate the concentration of solute adsorbed on the fiber surface and the intrapore concentration:

$\frac{\partial \mathrm{n}}{\partial \mathrm{t}}=\frac{\mathrm{qk}_{1}}{\mathrm{C}_{0}}\left(\mathrm{~N}_{0}-\mathrm{n}\right)-\mathrm{k}_{2} \mathrm{n}$

The deposition rate constant $k_{1}$ is second order in the forward direction and the detachment rate constant $k_{2}$ is first order in the backward direction. At equilibrium, equation (2) reduces to a Langmuir adsorption isotherm:

$\mathrm{n}=\frac{\mathrm{qk}^{*} \mathrm{~N}_{0}}{\mathrm{qk}^{*}+\mathrm{C}_{0}}$

\section{Model Equations for the Bulk Fluid Phase}

$$
u \frac{\partial c}{\partial z}+\frac{\partial c}{\partial t}+\frac{2(1-\varepsilon) k_{f}}{\varepsilon K R}(c-q)=D_{L} \frac{\partial^{2} c}{\partial z^{2}}
$$

Boundary conditions:

$$
\mathrm{uc}-\mathrm{D}_{\mathrm{L}} \frac{\partial \mathrm{c}}{\partial \mathrm{z}}=0 \quad \text { at } z=0
$$

$$
\frac{\partial \mathrm{c}}{\partial \mathrm{z}}=0 \quad \text { at } z=L
$$


Initial condition:

$\mathrm{c}=\mathrm{q}=\mathrm{C}_{0}$ and $\mathrm{n}=\mathrm{N}_{0}$ at $t=0$

The set of equations (1) to (7) are converted into the following dimensionless form using dimensionless variables defined in the list of nomenclature:

$$
\begin{aligned}
& \frac{\partial \mathrm{Q}}{\partial \tau}+\frac{1-\beta}{\beta} \mathrm{N}^{\prime} \frac{\partial \mathrm{N}}{\partial \tau}=\mathrm{Bi}(\mathrm{C}-\mathrm{Q}) \\
& \frac{\partial \mathrm{N}}{\partial \tau}=\mathrm{P}^{*}\left[\mathrm{Q}(1-\mathrm{N})-\left(\frac{\mathrm{N}}{\mathrm{k}^{*}}\right)\right] \\
& \frac{\partial \mathrm{C}}{\partial \tau}=\frac{1}{\operatorname{Pe}} \frac{\partial^{2} \mathrm{C}}{\partial \xi^{2}}-\frac{\partial \mathrm{C}}{\partial \xi}-\theta \mathrm{Bi}(\mathrm{C}-\mathrm{Q})
\end{aligned}
$$

Boundary conditions:

$$
\begin{aligned}
& \mathrm{C}-\frac{1}{\operatorname{Pe}} \frac{\partial \mathrm{C}}{\partial \xi}=0 \text { at } \xi=0 \\
& \frac{\partial \mathrm{C}}{\partial \xi}=0 \quad \text { at } \xi=1
\end{aligned}
$$

Initial condition:

$$
\mathrm{C}=\mathrm{Q}=\mathrm{N}=1 \quad \text { at } \tau=0
$$

\section{ORTHOGONAL COLLOCATION ON FINITE ELEMENTS (OCFE)}

The method of orthogonal collocation on finite elements (OCFE) is used to solve the system of partial differential equations (8) to (13). This method was first proposed by Carey and Finlayson (1975) to solve the stiff system of ordinary differential equations involving Thieles modulus. In this method, the axial domain is divided into small elements and then orthogonal collocation is applied within each element. Convergence of numerical solutions does not depend upon the number of collocation points, but rather on the number of elements to be taken in the domain of interest. The rate of convergence of OCFE is of $O\left(\max \left(\mathrm{h}_{\ell}^{2}\right)\right)$, where $\mathrm{h}_{\ell}$ is the length of the $\ell^{\text {th }}$ element. The effect of collocation points on the solution profile decreases as the number of collocation points increases from 5 . The convergence criterion of OCFE and details of choice of collocation points are given elsewhere (Arora et al., 2006b, 2006c; Onah, 2002).

\section{Numerical Simulation}

The OCFE is applied to the set of partial differential equations (PDE's) (8) to (13), which convert these PDE's into a set of differential algebraic equations (DAE's) as follows:

$$
\begin{aligned}
& \frac{\partial \mathrm{Q}_{\mathrm{j}}^{\ell}}{\partial \tau}+\frac{1-\beta}{\beta} \frac{\partial \mathrm{N}_{\mathrm{j}}^{\ell}}{\partial \tau}=\mathrm{Bi}\left(\mathrm{C}_{\mathrm{j}}^{\ell}-\mathrm{Q}_{\mathrm{j}}^{\ell}\right) \\
& j=2,3, \ldots, m \text { and } \ell=1,2,3, \ldots, r \\
& \frac{\partial \mathrm{N}_{\mathrm{j}}^{\ell}}{\partial \tau}=\mathrm{P}^{*}\left[\mathrm{Q}_{\mathrm{j}}^{\ell}\left(1-\mathrm{N}_{\mathrm{j}}^{\ell}\right)-\mathrm{k}^{*}-1 \mathrm{~N}_{\mathrm{j}}^{\ell}\right] \\
& j=2,3, \ldots, m \text { and } \ell=1,2,3, \ldots, r \\
& \frac{\partial \mathrm{C}_{\mathrm{j}}^{\ell}}{\partial \tau}=\frac{1}{\mathrm{Pe} \mathrm{h}_{\ell}^{2}} \sum_{\mathrm{i}=1}^{\mathrm{m}+1} \mathrm{~B}_{\mathrm{ji}} \mathrm{C}_{\mathrm{i}}^{\ell}-\frac{1}{\mathrm{~h}_{\ell}} \sum_{\mathrm{i}=1}^{\mathrm{m}+1} \mathrm{~A}_{\mathrm{ji}} \mathrm{C}_{\mathrm{i}}^{\ell}-\theta \mathrm{Bi}\left(\mathrm{C}_{\mathrm{j}}^{\ell}-\mathrm{Q}_{\mathrm{j}}^{\ell}\right) \\
& j=2,3, \ldots, m \text { and } \ell=1,2,3, \ldots, r \\
& \sum_{\mathrm{i}=1}^{1}-\frac{1}{\mathrm{Pe} \mathrm{h}_{1}} \sum_{\mathrm{i}=1}^{\mathrm{m}+1} \mathrm{~A}_{\mathrm{m}+1 \mathrm{i}} \mathrm{C}_{\mathrm{i}}^{\mathrm{r}}=0 . \\
& \mathrm{m}+1 \\
& \text { at } \xi=0
\end{aligned}
$$

The system of equations from (14) to (18) can be written as $\mathrm{DC}=\mathrm{MC}$, where $\mathrm{DC}$ represents the column matrix of time derivatives of $\mathrm{C}_{\mathrm{i}}^{\ell}$ (which is zero in case of algebraic equations), $\mathrm{M}$ is the coefficient matrix of collocation constants and $\mathrm{C}$ is the column matrix of unknown solution values at collocation points. Differential index refers to the number of differentiations require to convert the algebraic equations into differential equations. It is an important factor to measure the difficulty in numerically integrating the set of DAE's. The differential index of a given set of DAE's is 1 . Since the stiffness ratio is very high, the degree of stiffness is assumed to be the same for all the simulated cases. However, a detailed study of the differential index of DAE's and degree of stiffness can also be found in Caracotsios and Stewart (1995). The resulting stiff system of $r(3 m+1)+1$ DAE's is solved using MATLAB with the ode15s system solver. This 
system solver uses backward differentiation formula (BDF) to integrate the set of DAE's. The BDF is an implicit multi-step method which is used to solve the system of DAE's. This method is widely used to solve the system of DAE's instead of the method of lines because, in this method, algebraic equations are not converted into differential equations as in the method of lines and has a higher convergence rate than the method of lines. Here it should be made clear that this statement is not contradictory to the differential index because the method of BDF depends upon differential index and degree of stiffness. Complete description of the numerical technique is given elsewhere (Shampine and Gear, 1979; Lin et al., 1999). The block diagonal structure of DAE's after application of OCFE is given in Figure 2. In the present study, the domain of interest has been divided into 5 elements and 5 collocation points have been taken within each element, including boundary points 0 and 1 . The zeros of shifted Legendre polynomials have been taken as collocation points. The collocation points and their respective weight functions can be obtained from Arora et al. (2006b).

\begin{tabular}{|c|c|}
\hline \multicolumn{2}{|c|}{ M } \\
\hline B.C. at $\xi=0$ & \multirow{2}{*}{0} \\
\hline $\operatorname{xXXXXXXXXX}$ & \\
\hline \multicolumn{2}{|c|}{ Continuity Condition } \\
\hline \multirow{2}{*}{0} & 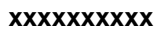 \\
\hline & B.C. at $\xi=1$ \\
\hline
\end{tabular}

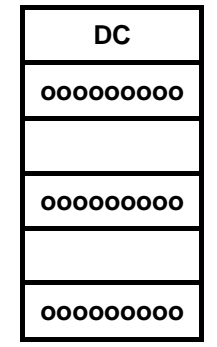

Figure 2: Block diagonal matrix structure for OCFE, the crosses arise after the application of orthogonal collocation within each element. The single column on the right hand side arises from the time derivatives of the dependent variable $C$ and the boxes represented by empty circles are for boundary conditions and continuity conditions.

\section{EXPERIMENTAL}

The stimulus-response experiments, using a step input, have been carried out in the displacement washing cell consisting of a vertical glass cylinder $110 \mathrm{~mm}$ high, $36.4 \mathrm{~mm}$ inside diameter, and closed at the lower end by a permeable septum (Figure 1). The fiber bed occupied the volume between the septum and a piston which could be inserted into the top of the cylinder. Both the piston and the septum were made permeable by 64 holes, $1 \mathrm{~mm}$ in diameter. In order to prevent losses of fine fibers from the bed, both piston and septum were covered with 45 mesh screen. During washing, direct observation of the pulp bed was possible. The experimental apparatus was similar to that used by Lee (1979).

Pulp beds were formed from a dilute suspension of unbeaten, unbleached kraft pulp in black liquor. In all runs, the beds were compressed to a final desired thickness of $30 \mathrm{~mm}$. The consistency of oven-dried pulp in the bed varied within the limits of 11.5 to $13.2 \%$. The average bed porosity was then between 0.65 and 0.70 . In order to characterize the pulp fibers used in the experiments, the physical properties of kraft pulp were determined as well. The Schopper-Riegler freeness had a value of 13 SR. The degree of delignification of pulp was expressed in terms of the kappa number, equal to 17 . The fiber length was determined from measurements in the wet state using the Kajaani FS-100 instrument. The weighted average length was $2.45 \mathrm{~mm}$, while the arithmetic average length was only $1.42 \mathrm{~mm}$. The ratio of the weighted to arithmetic average length, which is a measure of the polydispersity of fiber length, was then 1.7. The coarseness of pulp fibers had a value of $0.217 \mathrm{mg} / \mathrm{m}$. The effective specific volume of fibers had a value of $2.55 \mathrm{~cm}^{3} / \mathrm{g}$ and the specific surface of fibers was $4.17 \times 10^{5} \mathrm{~m}^{-1}$. Measurements of physical properties are described in detail in Potucek (1997).

To start the washing experiment, distilled water maintained at a temperature of about $20^{\circ} \mathrm{C}$ was distributed uniformly via the piston to the top of bed, approximating a step change in concentration. Depending on the permeability of the pulp bed formed in the washing cell, the wash liquid was forced through the pulp bed under a pressure drop of up to $7 \mathrm{kPa}$. The wash liquid superficial velocity varied in the range of 0.06 to $0.57 \mathrm{~mm} / \mathrm{s}$. Samples of the washing effluent, taking at different time intervals until the effluent was colourless, were analyzed for alkali lignin using an ultraviolet spectrophotometer operating at a wavelength of 295 $\mathrm{nm}$. The initial bed liquor concentration was $50 \mathrm{~g}$ of alkali lignin per liter.

\section{RESULTS AND DISCUSSION}

Even if the experimental conditions were strictly identical, the fiber bed was always different owing to the natural source of the material studied. The pulp bed, characterized as a fixed bed, consisted of compressible porous particles where geometrical 
similarity does not exist. With respect to the nature of the system studied, perfect plug flow displacement cannot be achieved for the dispersion due to inhomogeneities of the fiber bed, e.g., stagnant liquor regions or by-pass flows. Moreover, displacement washing of pulp fibers is affected by a number of phenomena occurring in porous medium, such as pore size distribution, different local porosity of the bed, geometrical properties of the fibers, including their swelling, intrinsic properties of miscible fluids, sorption, electrokinetic phenomena, and others.

In the washing process, no single factor affects the system, but rather these parameters depend on each other. The dimensionless Peclet number signifies a ratio of the convective to the diffusive transport mechanisms in the pulp bed. The Biot number is a measure of the relative importance of the external to internal mass transfer resistances when a solute is leached from fiber walls to the wash liquid flowing through inter-particle voids. While the Biot number depends upon interstitial wash liquid velocity, the Peclet number indicating a level of dispersion is influenced mainly by the inhomogeneities in the pulp bed, in which different local porosities lead to deviations from the plug flow, known as channeling or fingering. Potucek (1997) reported that the axial dispersion coefficient increases linearly with increasing wash liquid superficial velocity. Moreover, at low wash liquid superficial velocity below $1 \mathrm{~mm} / \mathrm{s}$, the wash liquid velocity appeared to have no effect on the shape of the breakthrough washing curve. However, interstitial velocity, as given by Darcy's law valid for streamline flow, depends upon bed porosity, which is determined with the help of effective specific volume of fibers and bed consistency. Hence, bed preparation plays an important role in the process of displacement washing and the determination of Peclet number and Biot number.

\section{Effect of Peclet Number on Exit Solute Concentration}

In Figure 3, the theoretical behavior of exit solute concentration profiles is shown for Biot number 10 and for different values of Peclet number. It is observed that, for $P e=10$, the solution profile take a long time to converge to steady-state condition as compared to $P e=30$. However, no significant effect is observed on solution profiles for $P e \geq 30$. AlJabari et al., (1994) have mentioned that, for displacement washing of the porous structure of particles, efficient washing operations can be achieved for $P e \leq 40$; however, washing operation depends upon the bed preparation and bed thickness.
In our case, bed thickness is comparatively small; however, the time taken by the solute to leach from the fiber surface is comparatively large, which contributes to small Peclet number. In the present case, the washing cell was prepared similar to that of Lee (1979) and Trinh et al. (1989), who have also found the Peclet number to be less than 30 . Hence, from this point of view our study agrees well with these investigators.

\section{Effect of Biot Number on Exit Solute Concentration}

Biot number signifies mass transfer resistance inside and on the surface of a body. In Figure 4, the theoretical behavior of exit solute concentration profiles is shown for $P e=30$ and for different values of Biot number. It is observed from this figure that no remarkable change occurs in solution profiles for $B i \geq 10$. For $B i=50$ and $B i=100$, solution profiles almost overlap each other. Mathematically, it may be due to the fact that an increase in Biot number reduces the effect of the dispersion term, which ultimately affects the solution profiles and, therefore, no significant change occurs for $B i \geq 10$. Raghvan and Ruthven (1983) have used the term film resistance parameter instead of Biot number and have shown that, under practical conditions of fixed bed adsorbers, $B i \leq 10$ is of practical importance because of large volume equilibrium constant.

\section{Effect of Pe and Bi on Exit Solute Concentration}

In Figure 5, solution profiles are plotted for different values of Peclet number and Biot number on the basis of experiments performed on a washing cell. Details of experiments are given in the experimental section. It is observed that better washing operations are achieved for $P e=21$ and $B i$ $=15$ as compared to $P e=8$ and $B i=2.3$. It is due to the fact that, for large Peclet number, axial dispersion is small, causing less back mixing. Similarly, for large Biot number, the $L / u$ ratio increases, as well as the film resistance mass transfer coefficient $k_{f}$. However, for small velocity profiles, the effect of $k_{f}$ is negligible. Both Peclet number and Biot number are affected by velocity profiles and also efficient washing operations can be achieved for small velocity profiles (Lee, 1979; Trinh et al., 1989). Therefore, from the theoretical point of view, breakthrough curves agree well with the theory that efficient washing operations can be achieved for large Peclet number and Biot number as compared to smaller ones. 


\section{Effect of $\mathrm{Pe}$ and $\mathrm{Bi}$ on Concentration of Solute Adsorbed on Fiber Surface}

In Figure 6, the theoretical behavior of solute concentration adsorbed on the fiber surface is shown for different values of Peclet number and Biot number. It is observed that, for $P e=11$ and $B i=5.5$, the retention time for the solute adsorbed on the fiber surface is higher compared to $P e=21$ and $B i=15$. It signifies that solute adsorbed on the fiber surface takes a long time to leach out from the fiber surface for small $P e$ and $B i$. It is due to the fact that, for small Peclet number, more back mixing occurs, causing more dispersion of solute and small Biot number reduces the equilibrium constant. Sridhar et al. (1994) also mentioned in their study that solute adsorbed on porous particles leaches out rapidly for Peclet number greater than 10 .

In Table 1, the relative error between

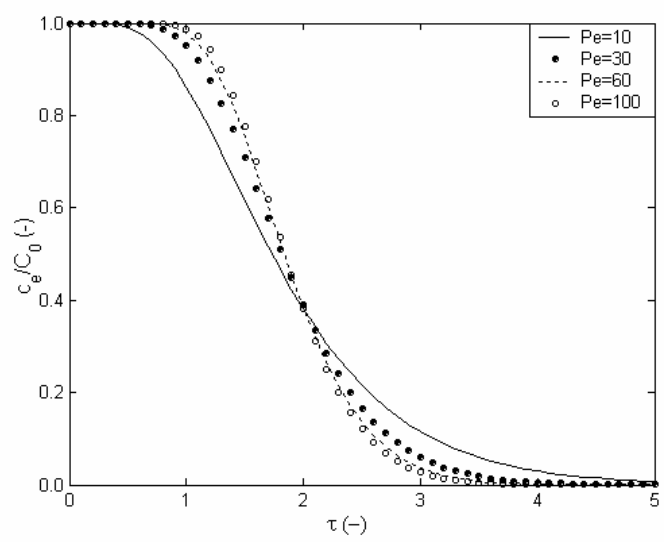

Figure 3: Behavior of concentration profiles for different values of Peclet number.

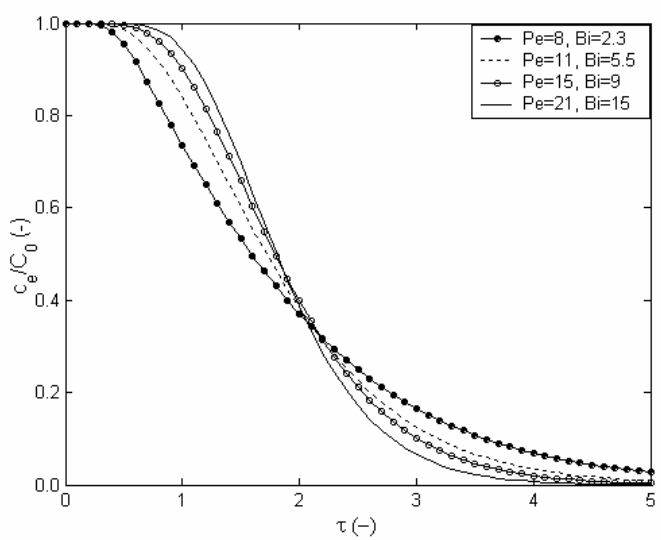

Figure 5: Behavior of solution profiles for different values of Peclet number and Biot number. experimental and calculated values is presented for two sets of washing runs. A good agreement is found between experimental and calculated values as the relative error is found to be less than $1 \%$ in both washing runs. It signifies the applicability of the model of the washing cell. It should be noted that, for washing runs I and II chosen for comparison with calculated exit solute concentrations, the bed consistency was 12.1 and $11.9 \%$, average bed porosity 0.690 and 0.684 , and wash liquid superficial velocity 0.245 and $0.380 \mathrm{~mm} / \mathrm{s}$, respectively. The initial concentration of solute, in our case alkali lignin, was $50 \mathrm{~kg} / \mathrm{m}^{3}$. The concentration of solute in fiber walls is $119 \mathrm{~kg} / \mathrm{m}^{3}$ and the average fiber radius in the wet state is $0.0225 \mathrm{~mm}$. The ratio of $k_{1} / k_{2}$ is calculated using the slope of $c$ and $c / n$ as discussed earlier in Arora et al. (2006a) and the film resistance mass transfer coefficient $k_{f}$ is found to be within the range of $(7.1-8.6) \times 10^{-7} \mathrm{~m} / \mathrm{s}$.

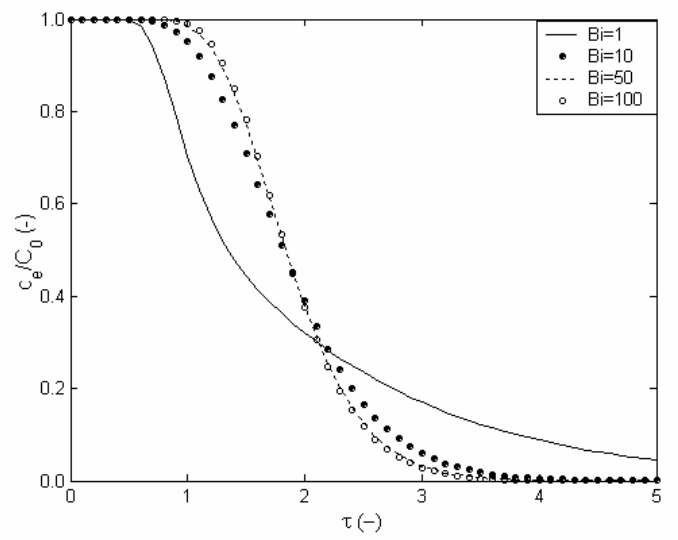

Figure 4: Behavior of concentration profiles for different values of Biot number.

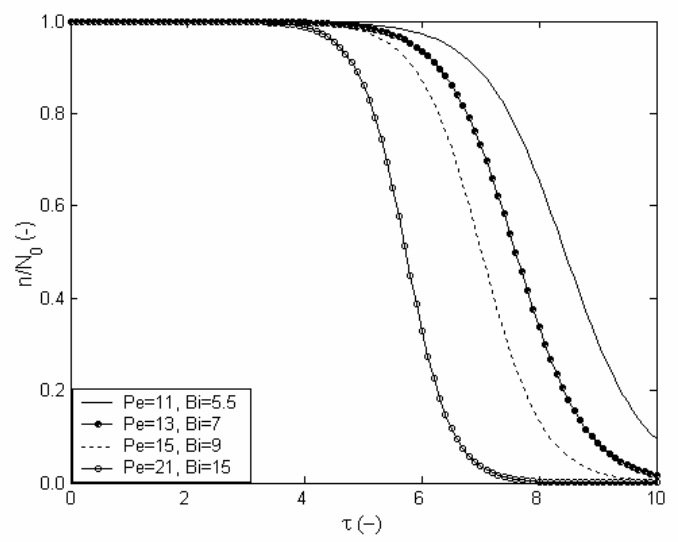

Figure 6: Behavior of solution profile for concentration of solute adsorbed on fiber surface for different values of Peclet number and Biot number. 
Table 1: Comparison of experimental and calculated values of dimensionless exit solute concentration

\begin{tabular}{|c|c|c|c|c|c|c|c|}
\hline \multicolumn{3}{|c|}{} & \multicolumn{4}{c|}{$\begin{array}{c}\text { For washing run II } \\
(\boldsymbol{P e}=\mathbf{1 4 . 1 3} \text { and } \boldsymbol{B i}=\mathbf{8 . 5})\end{array}$} \\
\hline$\tau$ & Experimental & Calculated & $\begin{array}{c}\text { Relative Error } \\
(\%)\end{array}$ & $\tau$ & Experimental & Calculated & $\begin{array}{c}\text { Relative Error } \\
(\%)\end{array}$ \\
\hline 0.343 & $9.5080 \times 10^{-1}$ & $9.5142 \times 10^{-1}$ & 0.0652 & 0.380 & $9.7070 \times 10^{-1}$ & $9.7060 \times 10^{-1}$ & 0.0103 \\
\hline 0.441 & $8.4810 \times 10^{-1}$ & $8.4652 \times 10^{-1}$ & 0.1863 & 0.494 & $8.1690 \times 10^{-1}$ & $8.1843 \times 10^{-1}$ & 0.1873 \\
\hline 0.563 & $6.5190 \times 10^{-1}$ & $6.4979 \times 10^{-1}$ & 0.3237 & 0.608 & $6.9180 \times 10^{-1}$ & $6.9152 \times 10^{-1}$ & 0.0405 \\
\hline 0.882 & $3.0720 \times 10^{-1}$ & $3.0796 \times 10^{-1}$ & 0.2474 & 0.912 & $3.8460 \times 10^{-1}$ & $3.8689 \times 10^{-1}$ & 0.5954 \\
\hline 2.107 & $9.7270 \times 10^{-3}$ & $9.7904 \times 10^{-3}$ & 0.6518 & 1.824 & $2.7110 \times 10^{-2}$ & $2.7220 \times 10^{-2}$ & 0.4058 \\
\hline 3.307 & $2.0310 \times 10^{-3}$ & $2.0378 \times 10^{-3}$ & 0.3348 & 3.686 & $1.3990 \times 10^{-3}$ & $1.3918 \times 10^{-3}$ & 0.5147 \\
\hline 4.802 & $8.1400 \times 10^{-4}$ & $8.1385 \times 10^{-4}$ & 0.0184 & 4.370 & $9.7070 \times 10^{-4}$ & $9.7972 \times 10^{-4}$ & 0.9292 \\
\hline
\end{tabular}

\section{Effect of $P e$ and $B i$ on Displacement Ratio}

Displacement ratio signifies the actual reduction in black liquor solids relative to the maximum possible reduction. Its formula is:

$\mathrm{DR}=\frac{\mathrm{C}_{0}-\mathrm{c}_{\mathrm{d}}}{\mathrm{C}_{0}-\mathrm{C}_{\mathrm{S}}}$

The average solute concentration $c_{d}$ is calculated from following equation:

$\mathrm{c}_{\mathrm{d}}=\mathrm{C}_{0} \sum_{\ell=1}^{\mathrm{r}} \mathrm{h}_{\ell} \sum_{\mathrm{i}=1}^{\mathrm{m}+1} \mathrm{w}_{\mathrm{i}} \mathrm{C}_{\mathrm{i}}^{\ell} ; \quad \ell=1,2,3, \ldots, r$

where the $w_{\mathrm{i}}$ 's are the weight functions of shifted Legendre polynomials and $h_{\ell}$ is the length of $\ell^{\text {th }}$ element. These $w_{\mathrm{i}}$ 's can be obtained from Arora et al. (2006b).

Kukreja et al. (1995) and Potucek (1997) have mentioned the dependence of average solute concentration on time and, therefore, displacement ratio is also dependent on time. It also depends upon the nature of the substance to be removed. In our case, lignin based solutes were analyzed in the exit stream of the filtrate. In breakthrough curves given by Figure 7, it is observed that a higher reduction in black liquor solids can be achieved for large Peclet number and Biot number. This is due to the fact that, for large Peclet number, retention of solute adsorbed on the fiber surface decreases because of less back mixing and dispersion of solute. Hence, more impurities adsorbed on the fiber surface can be removed within a shorter time interval and hence greater effectiveness of washing operations can be achieved.

\section{Effect of $P e$ and $B i$ on Efficiency}

Potucek (1997) has also shown the dependence of bed efficiency, $E$, on exit solute concentration, which depends upon time of washing, and hence the bed efficiency is also dependent on time. The bed efficiency may be defined by a relationship similar to the Murphree efficiency, which is often used for distillation trays, as:

$\mathrm{E}=\frac{\mathrm{c}_{\mathrm{e}}-\mathrm{C}_{\mathrm{S}}}{\mathrm{c}_{\mathrm{d}}-\mathrm{C}_{\mathrm{S}}}$

In the case of pure displacement, when perfect plug flow exists, the bed efficiency increases from one to infinity with increasing wash liquor ratio from 0 to 1 . On the other hand, for a perfectly mixed vessel, when the outlet solute concentration is equal to the concentration inside the bed, the efficiency is equal to unity. The removal of lignin from the pulp bed is accomplished via combined displacement and diffusion processes in our case of leaching of lignin from fiber walls. Both mechanisms, displacement, and leaching, occur simultaneously. Since the displacement mechanism prevails over leaching in the displacement washing of pulp fibers, a bed efficiency greater than unity should be achieved.

Bed efficiency is the key industrial factor that is highly affected by Peclet number and Biot number. In Figure 8, breakthrough curves are plotted for bed efficiency with respect to time for different values of Peclet number and Biot number. Significant changes have been observed in breakthrough curves for different Peclet number and Biot number. Exit and average solute concentration profiles do not change as significantly as bed efficiency. This is due to the fact that the net change in quantity of black liquor solids removed during the washing operation can be seen in efficiency, rather than in exit and average solute concentration. Therefore, efficiency of the equipment is observed from quantity of black liquor solids removed, which is determined from bed efficiency. It is obvious from Figure 8 that efficiency of the equipment increases with increasing Peclet number and Biot number. 


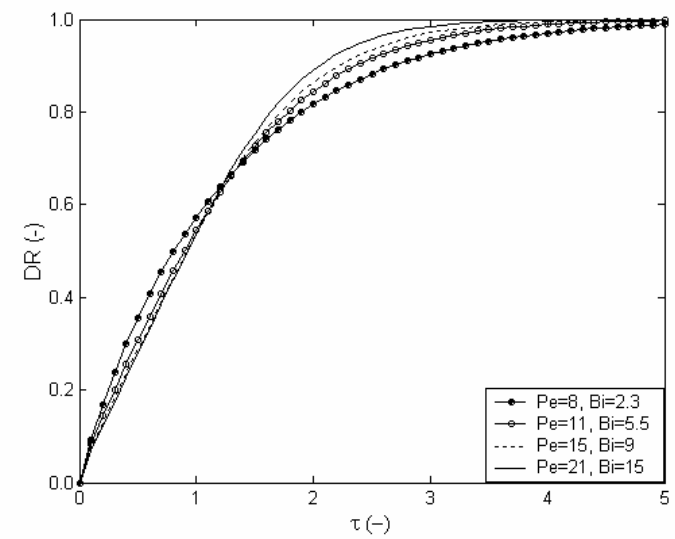

Figure 7: Behavior of displacement ratio for different Figure 8: Behavior of efficiency for different values of values of Peclet number and Biot number.

\section{CONCLUSIONS}

In this paper, a mathematical model for a displacement washing cell is proposed. The model includes both the Peclet number and Biot number. Peclet number controls axial dispersion of the fluid whereas Biot number controls the mass transfer resistances. It was observed that both Peclet number and Biot number affect the washing process. Experimental values of exit solute concentration were compared to the calculated ones and the relative error was found to be in the range of $\pm 1 \%$. It was also observed from breakthrough curves that bed efficiency is more highly affected by Peclet number and Biot number than exit solute concentration. It was also shown that efficiency of the equipment increases with Peclet number and Biot number. However, efficiency increases substantially only up to Peclet number 30 and Biot number 10.

\section{NOMENCLATURE}

$\begin{array}{ll}B i & \text { Biot number, }=\frac{\mathrm{k}_{\mathrm{f}} \mathrm{L} \beta}{\mathrm{KRu}} \\ c & \begin{array}{l}\text { Concentration of solute in } \\ \text { liquor }\end{array} \\ c_{d} & \begin{array}{l}\text { Average solute } \\ \text { concentration in packed bed }\end{array} \\ c_{e} & \text { Exit solute concentration } \\ C & \text { Dimensionless concentration } \\ C_{S} & \text { Solute concentration in wash } \\ C_{0} & \text { liquor } \\ & \text { Initial solute concentration }\end{array}$

dimensionless

$\begin{array}{ll}\mathrm{kg} / \mathrm{m}^{3} & q \\ & \\ \mathrm{~kg} / \mathrm{m}^{3} & Q \\ & R \\ \mathrm{~kg} / \mathrm{m}^{3} & t \\ =c / C_{0} & u \\ \mathrm{~kg} / \mathrm{m}^{3} & \\ \mathrm{~kg} / \mathrm{m}^{3} & \end{array}$

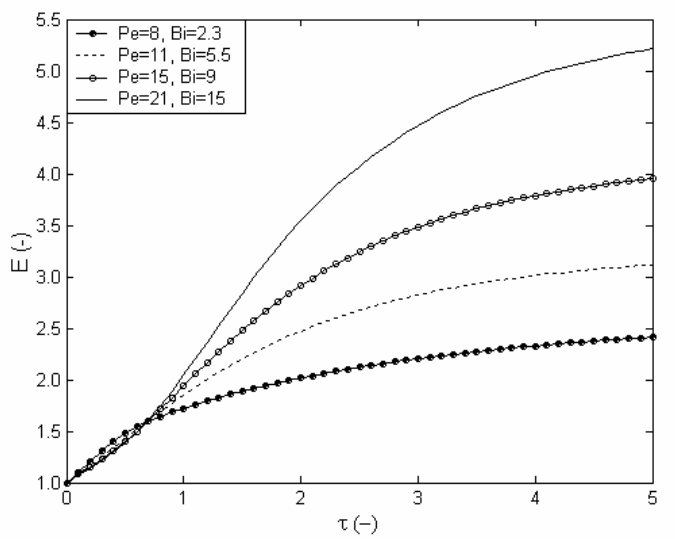

Peclet number and Biot number.
$D_{L}$

$D R$

E

$k_{f}$

$k_{1}$

$k_{2}$

$k^{*}$

$k^{*}$
$K$

$L$

$n$

$N$

$N_{0}$

\section{$N^{\prime}$}

$P^{*}$

Pe

$q$

$Q$

$R$

$u$

$z$
Axial dispersion coefficient

$\mathrm{m}^{2} / \mathrm{s}$

Displacement ratio

Bed efficiency

Film resistance mass

transfer coefficient

$\mathrm{m} / \mathrm{s}$

Mass transfer coefficient

$1 / \mathrm{s}$

for solute adsorption

Mass transfer coefficient

$1 / \mathrm{s}$

for solute desorption

Dimensionless parameter

Volume equilibrium

constant

Thickness of the bed

Concentration of solute

adsorbed on the fibers

Dimensionless concentration

of solute adsorbed on fibers

Initial concentration of

solute adsorbed on the fibers

Dimensionless parameter

Dimensionless parameter

Peclet number,

ntrapore solute

concentration

Dimensionless intrapore

solute concentration

Pore radius of fibers

$=\mathrm{k}_{1} / \mathrm{k}_{2}$

dimensionless

$$
\begin{aligned}
& \underset{\mathrm{kg} / \mathrm{m}^{3}}{\mathrm{~m}} \\
& =n / N_{0} \\
& \mathrm{~kg} / \mathrm{m}^{3} \\
& =N_{0} / C_{0} \\
& =\mathrm{k}_{1} \mathrm{~L} / \mathrm{u} \\
& =\mathrm{uL} / \mathrm{D}_{\mathrm{L}}
\end{aligned}
$$

dimensionless

$\mathrm{I} \mathrm{kg} / \mathrm{m}^{3}$

$=q / C_{0}$

Time

m

Interstitial wash liquid

velocity through bed

Distance from point of

introduction of solvent $\mathrm{m} / \mathrm{s}$

$\mathrm{m}$ 


\section{Greek Symbols}

$\begin{array}{llr}\beta & \text { Fiber porosity } & \begin{array}{r}\text { dimensionless } \\ \text { dimensionless } \\ =2(1-\varepsilon) / \varepsilon\end{array} \\ \theta & \begin{array}{l}\text { Porosity of bed } \\ \text { Dimensionless parameter, }\end{array} & \begin{array}{r}\text { dimensionless } \\ =\mathrm{z} / \mathrm{L}\end{array} \\ \xi & \begin{array}{l}\text { Dimensionless axial } \\ \text { distance, }\end{array} & \begin{array}{r}\text { dimensionless } \\ =\mathrm{tu} / \mathrm{L}\end{array} \\ \tau & \text { Dimensionless time, } & \text { dimensionless }\end{array}$

\section{REFERENCES}

Al-Jabari, M., Van Heiningen, A. R. P. and Van De Ven T. G. M., Modeling the flow and the deposition of fillers in packed bed of pulp fibers, Journal of Pulp and Paper Science, 20, No. 9, pp. J249-J253 (1994).

Arora, S., Dhaliwal, S. S. and Kukreja, V. K., Simulation of washing of packed bed of porous particles by orthogonal collocation on finite elements, Computers and Chemical Engineering, 30, No. 6-7, pp. 1054-1060 (2006a).

Arora, S., Dhaliwal, S. S. and Kukreja, V. K., A computationally efficient technique for solving two point boundary value problems in porous media, Applied Mathematics and Computation, 183, No. 2, pp. 1170-1180 (2006b).

Arora, S., Dhaliwal, S. S. and Kukreja, V. K., On the convergence behavior of the method of orthogonal collocation on finite elements, International Congress of Mathematicians, Madrid, Spain (2006c).

Balasubramanian, V., Jayaraman, G. and Iyenger, S. R. K., Effect of secondary flowsmon contaminant dispersion with weak boundary absorption, Applied Mathematical Modelling, 21, pp. 275285 (1997).

Bhatia, S. K., Modeling the pore structure of coal, AIChE Journal, 33, No.10, pp.1707-1718 (1987).

Caracotsios, M., and Stewart, W. E. Sensitivity analysis of initial boundary value problems with mixed PDE's and algebraic equations: Applications to chemical and biochemical systems, Computers and Chemical Engineering, 19, No. 9, pp. 1019-1030 (1995).

Carey, G. F. and Finlayson, B. A., Orthogonal collocation on finite elements, Chemical Engineering and Science, 30, pp. 587-596 (1975).

Ding, L. P. and Bhatia, S. K. Analysis of multicomponent adsorption kinetics on activated carbon, AIChE, 49, No.5 pp. 883-895 (2003).

Eriksson, G., Rasmuson, A., and Theliander, H., Displacement washing of lime mud : tailing effects, Seperation Technology, 6, pp. 201-210 (1996).

Immanuel, C. D., Doyle III, F. J., Cordeiro, C. F. and Sundaram, S. S., Population balance PSD model for emulsion polymerization with steric stabilizers, AIChE, 49, No. 6, pp. 1392-1404 (2003).

Kukreja, V. K., Ray, A. K., Singh, V. P., and Rao, N. J., A mathematical model for pulp washing in different zones of a rotary vaccum filter, Indian Chemical Engineer Section A, 37, No. 3, pp. 113124 (1995).

Lee, P. F., Optimizing the displacement washing of pads of wood pulp fibers, Tappi, 62, No. 9, pp. 75-78 (1979).

Lin, Y. H., Chang, H. Y. and Adomaitis, R. A., MWR tools: Alibrary of weighted residual method calculations, Computers and Chemical Engineering, 23, No. 8, pp. 1041-1061 (1999)

Liu, F. and Bhatia, S. K., Application of PetrovGalerkin methods to transient boundary value problems in chemical engineering: Adsorption with steep gradients in bidisperse solids, Chemical Engineering Science, 56, pp. 3727-3735 (2001).

Onah, S. E., Asymptotic behavior of the Galerkin and the finite element collocation methods for a parabolic equation, Applied Mathematics and Computation, 127, pp. 207-213 (2002).

Potucek, F., Washing of pulp bed, Collection of Czechoslovak Chemical Communications, 62, pp. 626 - 644 (1997).

Raghvan, N. S. and Ruthven, D. M., Numerical simulation of a fixed bed adsorption column by the method of orthogonal collocation, AIChE, 29, No. 6, pp. 922-925 (1983).

Shampine, L. and Gear, C., A user's view of solving stiff ordinary differential equations, SIAM Review, 21, pp. 1-17 (1979).

Sharma, M. M. and Yortsos, Y. C., Transport of particulate suspensions in porous media: Model formulation, AIChE, 33, No. 10, pp. 1636-1643 (1987).

Sridhar P., Sastri, N. V. S., Modak, J. M. and Mukherjee, A. K., Mathematical simulation of bioseparation in an affinity packed column. Chemical Engineering and Technology, 17, pp. 422-429 (1994).

Trinh, D. T., Poirier, N. A., Crotogino, R. H. and Douglas, W. J. M., Displacement washing of wood pulp - an experimental study, Journal of Pulp and Paper Science, 15, No. 1, pp. J28-J35 (1989). 